\title{
Relationship Between Cerebral Microbleeds and Aspirin Use Regarding White Matter Hyperintensity Volume
}

\author{
Chi Kyung Kim, MD, $\mathrm{PhD}^{*}, 1$ (D); Jung-Hoon Han, $\mathrm{MD}^{*}{ }^{* 1}$ (D); Han-Yeong Jeong, $\mathrm{MD}^{\dagger}$ (D); Kyungmi Oh, MD, $\mathrm{PhD}^{*}$ (D); \\ Jin-Ho Park, MD, MPH, PhD ${ }^{\ddagger} \mathbb{D}$; Hyung-Min Kwon, MD, PhD ${ }^{\mathbb{Q}} \mathbb{D}$ \\ Department of Neurology, Korea University Guro Hospital, Korea University College of Medicine*, Seoul; Department of Neurology, Seoul \\ National University Hospital ${ }^{\dagger}$, Seoul; Department of Family Medicine, Seoul National University Hospital ${ }^{\ddagger}$, Seoul; Department of Neurology, \\ Seoul National University-Seoul Municipal Government Boramae Medical Center ${ }^{\S}$, Seoul, Korea
}

Background: The association between antiplatelet use and cerebral microbleeds (CMBs) remains controversial. Herein, we investigated the effects of aspirin use on CMBs according to white matter hyperintensity (WMH) volume.

Methods: CMBs were detected on gradient-recalled echo magnetic resonance imaging, and the volume of WMH was measured quantitatively. Information about aspirin use was obtained using a structured questionnaire, and other clinical and laboratory variables were investigated. The association between aspirin use and the presence of CMBs was evaluated according to WMH volume quartiles.

Results: Among 1,642 neurologically asymptomatic subjects, 69 (4.2\%) had CMBs and 126 (8\%) had taken aspirin. The mean volume of WMH was $2.7 \mathrm{~mL}$, and the average age was 56 years. The proportion of aspirin use did not differ between the two groups with or without CMBs $(13 \%$ vs. $7 \%$, respectively, $p=0.11)$. In the highest quartile for $\mathrm{WMH}$ volume, the prevalence of CMBs and aspirin use increased compared with the lowest quartiles (10\% vs. $1 \%$ for CMBs, and $11 \%$ vs. $4 \%$ for aspirin use). However, in each quartile of WMH volume, the proportion of aspirin use did not differ between subjects with and without CMBs. Chronological age and hypertension were associated with the presence of CMBs in both univariate and multivariate analyses after adjusting for WMH volume, but aspirin use did not increase the prevalence of CMBs in logistic regression analysis.

Conclusion: Using WMH volume as a radiological marker for the vulnerable brain could not assist in distinguishing high-risk subjects for an increased prevalence of CMBs with regard to aspirin use.

J Neurosonol Neuroimag 2021;13(1):1-6

Key Words: Aspirin; Cerebral small vessel diseases; Microbleeds
Received: November 10, 2020 Revised: February 2, 2021 Accepted: February 12, 2021

Correspondence: Hyung-Min Kwon, MD, PhD Department of Neurology, Seoul National University-Seoul Municipal Government Boramae Medical Center, 20 Boramae-ro 5-gil, Dongjak-gu, Seoul 07061, Korea Tel: +82-2-870-2475

Fax: $+82-2-831-2826$

E-mail: hmkwon@snu.ac.kr

Jin-Ho Park, MD, MPH, PhD

Department of Family Medicine, Seoul National University Hospital, 101 Daehak-ro, Jongro-gu, Seoul 03080, Korea Tel: +82-2-2072-0865

Fax: $+82-2-766-3296$

E-mail:kkolzzio@gmail.com

${ }^{1}$ The two authors contributed equally to the work.

\section{INTRODUCTION}

Cerebral microbleeds (CMBs) are well-known markers for cerebral small vessel disease on magnetic resonance imaging (MRI). ${ }^{1}$ Compared with other imaging markers for subclinical arteriolopathy in the brain, CMBs reflect a hemorrhage-prone state and are usually more associated with hypertension. ${ }^{2}$ Because of its bleeding tendency, aspirin use has been suspected as a risk factor for CMBs. Although antiplatelet use has not been associated with the presence of CMBs in a few clinical studies, ${ }^{3,4}$ a recent meta-analysis showed a potential association between antiplatelet use and CMBs in stroke patients, ${ }^{5}$ but not in stroke-free patients. ${ }^{6}$ However, because of the heterogeneity of the study population and different bleeding risks in individual subjects, this association remains controversial.,

White matter hyperintensity (WMH) is another imag- 
ing marker for cerebral small vessel disease and more closely related to chronic ischemic damage in the brain than CMBs. ${ }^{9}$ Interestingly, WMH was related to not only future ischemic events but also the occurrence of intracerebral hemorrhage because of the frailty of small vessels in the brain by chronic ischemic insults. ${ }^{10,11}$ Because some clinicians prescribe antiplatelet drugs in asymptomatic subjects with advanced WMH to prevent additional ischemic insult, it would be interesting to evaluate the effects of aspirin use on the presence of CMBs according to WMH volume.

\section{SUBJECTS AND METHODS}

We initially recruited a consecutive series of 1,646 neurologically asymptomatic subjects who visited Seoul National University Hospital Health Promotion Center for routine health check-ups between January 2006 and December 2011 in different centers from the previous study. ${ }^{3}$ Asymptomatic subjects were defined as those who had not complained of any neurological symptoms or signs. Four subjects were excluded because they did not undergo adequate MRI; finally, 1,642 subjects' data were included in the analysis. Clinical information was obtained through personal interviews, and a physical examination was performed by a trained physician.
This study was approved by the Institutional Review Board of Seoul National University Hospital (IRB No. 1502-026-647).

Hypertension was defined as a high systolic blood pressure $(\geq 140 \mathrm{mmHg}$ ), a high diastolic blood pressure $(\geq 90 \mathrm{mmHg}$ ), or the use of antihypertensive drugs when the subject visited. Subjects were considered to have diabetes if their fasting glucose level was $\geq 126 \mathrm{mg} / \mathrm{dL}$ or they reported the current use of antidiabetic agents. Hyperlipidemia was defined as a total cholesterol $>240$ $\mathrm{mg} / \mathrm{dL}$ or use of cholesterol-lowering drugs when the subjects were enrolled. Information about aspirin use, the presence of concomitant coronary artery disease, and cigarette smoking were obtained using a structured questionnaire. Total cholesterol, high-density lipoprotein cholesterol, and triglyceride levels were measured after fasting for $\geq 8$ hours.

CMBs were defined as a well-demarcated focal lesion less than $5 \mathrm{~mm}$ in diameter with a low signal on gradient-recalled echo (GRE) MRI. WMH volume was analyzed by a stroke neurologist (H-YJ) using the previously defined method. ${ }^{12}$ MRI was performed at 1.5-Tesla field strength (Magnetom SONATA, Siemens, Munich, Germany or SIGNA, GE Healthcare, Milwaukee, WI, USA). The imaging protocol consisted of T1-weighted (repetition time/echo time $=500 / 11 \mathrm{~ms}$ ), T2-weighted (repetition time/echo time $=5,000 / 127 \mathrm{~ms}$ ), and fluid-at-

TABLE 1. Baseline characteristics according to the presence of cerebral microbleeds

\begin{tabular}{|c|c|c|c|c|}
\hline & \multirow{2}{*}{ Total $(n=1,642)$} & \multicolumn{2}{|c|}{ Cerebral microbleeds } & \multirow{2}{*}{$p$-value } \\
\hline & & Yes $=69$ & $\mathrm{No}=1,573$ & \\
\hline Age (years) & $56 \pm 9$ & $63 \pm 8$ & $55 \pm 9$ & $<0.01$ \\
\hline Sex (male) & $861(52)$ & $40(58)$ & $821(52)$ & 0.39 \\
\hline Hypertension (yes) & $449(27)$ & $35(51)$ & $414(26)$ & $<0.01$ \\
\hline Diabetes (yes) & $181(11)$ & $15(22)$ & $166(11)$ & $<0.01$ \\
\hline Hyperlipidemia (yes) & $200(12)$ & $10(15)$ & $190(12)$ & 0.57 \\
\hline Coronary artery disease (yes) & $44(3)$ & $3(4)$ & $41(3)$ & 0.38 \\
\hline Smoking (yes) & $713(43)$ & $29(42)$ & $684(44)$ & 0.90 \\
\hline Total cholesterol (mg/dL) & $199 \pm 35$ & $202 \pm 34$ & $199 \pm 35$ & 0.51 \\
\hline Triglyceride (mg/dL) & $123 \pm 75$ & $126 \pm 60$ & $123 \pm 76$ & 0.69 \\
\hline HDL-cholesterol (mg/dL) & $54 \pm 14$ & $53 \pm 12$ & $54 \pm 14$ & 0.48 \\
\hline Aspirin use (yes) & $126(8)$ & $9(13)$ & $117(7)$ & 0.11 \\
\hline The volume of WMH (mL) & $2.7 \pm 5.8$ & $9.9 \pm 17.4$ & $2.4 \pm 4.4$ & $<0.01$ \\
\hline
\end{tabular}

Values are presented as number (\%) or mean \pm standard deviation.

$\mathrm{HDL}$; high-density lipoprotein, $\mathrm{WMH}$; white matter hyperintensity. 
tenuated inversion recovery imaging (repetition time/ echo time $=8,800 / 127 \mathrm{~ms}$; inversion time $=2,250 \mathrm{~ms}$ ). Twenty-four transaxial slices per scan were selected with a 5-mm slice thickness and a 1-mm interslice gap. T2*-weighted GRE MRI was performed in the axial plane with the following parameters: repetition time/ echo time, 500/15 ms; flip angle, $26^{\circ}$; slice thickness, $5 \mathrm{~mm}$; and gap width, $1 \mathrm{~mm}$ ). MR images were independently evaluated by two stroke neurologists (J-SL and $\mathrm{H}-\mathrm{MK})^{13}$

We performed Student's t-test or the Wilcoxon's rank-sum test for continuous variables and the $\chi^{2}$ test

TABLE 2. Baseline characteristics according to the presence of aspirin use status

\begin{tabular}{|lccc|}
\hline & \multicolumn{2}{c}{ Aspirin use } & \multirow{2}{*}{-value } \\
\cline { 2 - 3 } & Yes=126(7.2) & No=1,516(92.3) & $<0.01$ \\
\hline Age (years) & $60 \pm 8$ & $55 \pm 9$ & 0.07 \\
Sex (male) & $76(60)$ & $785(52)$ & $<0.01$ \\
Hypertension (yes) & $79(63)$ & $370(24)$ & $<0.01$ \\
Diabetes (yes) & $33(26)$ & $148(10)$ & $<0.01$ \\
Hyperlipidemia (yes) & $46(37)$ & $154(10)$ & $<0.01$ \\
Coronary artery disease (yes) & $16(13)$ & $28(2)$ & 0.32 \\
Smoking (yes) & $60(48)$ & $653(43)$ & 0.02 \\
Total cholesterol (mg/dL) & $192 \pm 37$ & $199 \pm 34$ & 0.54 \\
Triglyceride (mg/dL) & $118 \pm 60$ & $123 \pm 76$ & 0.25 \\
HDL-cholesterol (mg/dL) & $53 \pm 13$ & $54 \pm 13$ & 0.09 \\
CMBs & $9(7)$ & $60(4)$ & 0.03 \\
The volume of WMH (mL) & $4.3 \pm 8.3$ & $2.6 \pm 5.5$ & \\
\hline
\end{tabular}

Values are presented as number (\%) or mean \pm standard deviation.

HDL; high-density lipoprotein, CMB; cerebral microbleeds, WMH; white matter hyperintensity.

TABLE 3. Variables according to the quartiles of volumes of white matter hyperintensity

\begin{tabular}{|c|c|c|c|c|c|}
\hline & & Quartiles of & VMH volume & & \\
\hline & $\begin{array}{c}\mathrm{Q}_{1}(\leq 0.3 \mathrm{~mL}) \\
(\mathrm{n}=452)\end{array}$ & $\begin{array}{c}\mathrm{Q}_{2}(>0.3 \text { and } \leq 1.2 \\
\mathrm{mL})(\mathrm{n}=397)\end{array}$ & $\begin{array}{c}\mathrm{Q}_{3}(>1.2 \text { and } \leq 2.8 \\
\mathrm{mL})(\mathrm{n}=385)\end{array}$ & $\begin{array}{c}\mathrm{Q}_{4}(>2.8 \mathrm{~mL}) \\
(\mathrm{n}=408)\end{array}$ & $p$-value \\
\hline Age (years) & $51 \pm 8$ & $53 \pm 8$ & $57 \pm 8$ & $62 \pm 8$ & $<0.01$ \\
\hline Sex (male) & $239(53)$ & $200(50)$ & $204(53)$ & $218(53)$ & 0.73 \\
\hline Hypertension (yes) & $75(17)$ & $88(22)$ & $117(30)$ & $169(41)$ & $<0.01$ \\
\hline Diabetes (yes) & $24(5)$ & $37(9)$ & $48(13)$ & $72(18)$ & $<0.01$ \\
\hline Hyperlipidemia (yes) & $45(10)$ & $56(14)$ & $45(12)$ & $54(13)$ & 0.26 \\
\hline Coronary artery disease (yes) & $6(1)$ & $11(3)$ & $12(3)$ & $15(4)$ & 0.03 \\
\hline Smoking (yes) & $210(47)$ & $168(42)$ & $167(43)$ & $168(41)$ & 0.02 \\
\hline Total cholesterol (mg/dL) & $198 \pm 35$ & $199 \pm 34$ & $202 \pm 35$ & $199 \pm 37$ & 0.25 \\
\hline Triglyceride (mg/dL) & $124 \pm 72$ & $122 \pm 83$ & $121 \pm 76$ & $122 \pm 71$ & 0.88 \\
\hline HDL-cholesterol (mg/dL) & $54 \pm 13$ & $53 \pm 13$ & $55 \pm 14$ & $55^{ \pm 14}$ & 0.43 \\
\hline Aspirin use (yes) & $16(4)$ & $33(8)$ & $33(9)$ & $44(11)$ & $<0.01$ \\
\hline The presence of CMBs (yes) & $5(1)$ & $10(3)$ & $13(3)$ & $41(10)$ & $<0.01$ \\
\hline
\end{tabular}

Values are presented as number (\%) or mean \pm standard deviation.

WMH; white matter hyperintensity, Q; quartile, HDL; high-density lipoprotein, CMB; cerebral microbleeds. 
or Fisher's exact test for categorical variables. Spearman's nonparametric correlation analysis was used to evaluate trends according to WMH volume quartiles. We calculated the odds ratios (ORs) and 95\% confidence intervals (CIs) of the presence of CMBs according to the groups of aspirin use using binary logistic regression analyses. Multivariate analyses were performed after adjustment for possible confounding factors of age, sex, hypertension, diabetes, smoking, aspirin use, and WMH volume. Interrater agreement was estimated using the $\kappa$ statistic for CMB evaluation in the image. All statistical analyses were performed using IBM SPSS Statistics 21 (IBM Co., Armonk, NY, USA), and twotailed probability values $<0.05$ were considered significant.

\section{RESULTS}

Among the 1,642 subjects, 69 (4.2\%) had CMBs and $126(8 \%)$ had taken aspirin. The mean WMH volume was $2.7 \pm 5.8 \mathrm{~mL}$ (range $0-107 \mathrm{~mL}$ ). The mean patient age was $56 \pm 9$ years (range $24-83$ years). Interrater agreement for $\mathrm{CMB}$ evaluation in the image was moderate $(\kappa=0.83)$. With regard to CMB occurrence, hypertension (51\% vs. $26 \%, p<0.01$ ) and diabetes ( $22 \%$ vs. $11 \%, p<0.01$ ) were significantly more prevalent, while age $(63 \pm 8$ vs. $55 \pm 9$ years, p<0.01), and WMH volume (9.9 \pm 17.4 vs. $2.4 \pm 4.4 \mathrm{~mL}$, $p<0.01$ ) were significantly higher in subjects with CMBs (Table 1). There was no significant intergroup difference in aspirin use (13\% vs. $7 \%$, respectively, $p=0.11$ ). Significantly more subjects had hypertension ( $63 \%$ vs. $52 \%$, p<0.01), diabetes (26\% vs. $10 \%$, $p<0.01$ ), hyperlipidemia ( $37 \%$ vs. $10 \%, p<0.01$ ), coronary artery disease ( $13 \%$ vs. $2 \%$, $p<0.01)$, older age ( $60 \pm 8$ vs. $55 \pm 9$, $p<0.01)$, higher $\mathrm{WMH}$ volume ( $4.3 \pm 8.3$ vs. $2.6 \pm 5.5 \mathrm{~mL}, p=0.03$ ), and lower total cholesterol level (192 \pm 37 vs. $199 \pm 34, p=0.02)$ in aspirin users (Table 2).

According to WMH volume quartiles, higher WMH volume was associated with older age and the presence of hypertension and diabetes (Table 3). The proportions of CMBs and aspirin use were higher in the highest quartile for WMH volume than in the lowest quartile (10\% vs. $1 \%$ for CMBs, and $11 \%$ vs. $4 \%$ for aspirin use).

In each quartile of WMH volume, the proportions of aspirin use did not differ according to CMBs (Table 4). In the univariate analysis, increased age, presence of hypertension and diabetes, and higher WMH volume were significantly associated with the presence of CMBs, but aspirin use did not increase the OR significantly for the presence of CMBs (Table 5; crude OR 1.87; 95\% CI 0.90-3.86). In the multivariate logistic regression analysis, age, hypertension, and increasing WMH volume were still associated with the presence of CMBs. As in the univariate analysis, aspirin use did not

TABLE 5. Logistic regression model for the presence of cerebral microbleeds

\begin{tabular}{|lcc|}
\hline & \multicolumn{2}{c|}{$\begin{array}{c}\text { The presence of cerebral } \\
\text { microbleeds }\end{array}$} \\
\cline { 2 - 3 } & $\begin{array}{c}\text { Crude OR } \\
(95 \% \mathrm{Cl})\end{array}$ & $\begin{array}{c}\text { Adjusted OR } \\
(95 \% \mathrm{Cl})\end{array}$ \\
\hline Age (per 1 year) & $1.10(1.07-1.13)$ & $1.06(1.03-1.10)$ \\
Sex (male) & $1.26(0.78-2.06)$ & $0.75(0.37-1.49)$ \\
Hypertension (yes) & $2.88(1.77-4.68)$ & $1.79(1.05-3.06)$ \\
Diabetes (yes) & $2.35(1.30-4.27)$ & $1.47(0.78-2.79)$ \\
Smoking (yes) & $0.94(0.58-1.54)$ & $0.79(0.39-1.58)$ \\
Aspirin use (yes) & $1.87(0.90-3.86)$ & $0.93(0.41-2.09)$ \\
WMH volume (per 1 mL) & $1.09(1.06-1.13)$ & $1.06(1.03-1.09)$ \\
\hline
\end{tabular}

OR; odd ratio, $\mathrm{Cl}$; confidence interval, $\mathrm{WMH}$; white matter hyperintensity.

TABLE 4. The proportion of aspirin use in groups with or without CMBs according to quartiles of WMH volume

\begin{tabular}{|c|c|c|c|c|c|c|c|c|}
\hline & \multicolumn{2}{|c|}{ WMH volume Q1 } & \multicolumn{2}{|c|}{ WMH volume Q2 } & \multicolumn{2}{|c|}{ WMH volume Q3 } & \multicolumn{2}{|c|}{ WMH volume $\mathrm{Q}_{4}$} \\
\hline & $\begin{array}{l}\text { CMB (-) } \\
(n=447)\end{array}$ & $\begin{array}{c}\mathrm{CMB}(+) \\
(n=5)\end{array}$ & $\begin{array}{l}\text { CMB }(-) \\
(n=387)\end{array}$ & $\begin{array}{c}\mathrm{CMB}(+) \\
(n=10)\end{array}$ & $\begin{array}{l}\text { CMB }(-) \\
(n=372)\end{array}$ & $\begin{array}{c}\mathrm{CMB}(+) \\
(n=13)\end{array}$ & $\begin{array}{l}\text { CMB (-) } \\
(n=367)\end{array}$ & $\begin{array}{c}\mathrm{CMB}(+) \\
(\mathrm{n}=41)\end{array}$ \\
\hline Aspirin use (yes) & $16(3.6)$ & $\circ(0)$ & $31(8)$ & $2(20)$ & $32(8.6)$ & $1(7 \cdot 7)$ & $38(10.4)$ & $6(14.6)$ \\
\hline$p$-value & \multicolumn{2}{|c|}{1.0} & \multicolumn{2}{|c|}{0.20} & \multicolumn{2}{|c|}{1.0} & \multicolumn{2}{|c|}{0.42} \\
\hline
\end{tabular}

Values are presented as number (\%).

CMB; cerebral microbleeds, WMH; white matter hyperintensity, Q; quartile. 
increase and was not related to the presence of CMBs (Table 5).

\section{DISCUSSION}

In this study, aspirin use was not associated with the presence of CMBs, even in patients with advanced WMH and more prevalent CMBs. Isolated cerebral $\mathrm{WMH}$ is not an indication for antiplatelets including aspirin. Nevertheless, several physicians prefer to prescribe antiplatelet therapy for patients with WMH who have multiple vascular risk factors such as hypertension, diabetes, and hyperlipidemia. Our data suggest that in neurologically healthy patients, this type of prescription may not be appropriate with regard to the existence of CMBs.

In the previous meta-analysis, the detection of CMBs was related to antiplatelet use in the stroke population, which can be considered to have compromised and vulnerable background brain structure. ${ }^{6}$ In the context of this result, we postulated that when WMH, which also represents chronic brain damage and susceptibility to future stroke, increases in the non-stroke population, aspirin use may be related to CMBs as in the stroke population. Although our results showed a negative relationship between aspirin use and CMB occurrence in relation to WMH, a strong correlation between WMH and CMBs, which share a common pathophysiology of cerebral small vessel diseases such as hypertensive arteriolopathy or cerebral amyloid angiopathy, ${ }^{9}$ was consistently found in our study.

Compared with our previous study of the elderly population from a different center, ${ }^{3}$ the prevalence of CMBs was lower in this study because we included an all-age population. Therefore, chronological age and hypertension were clearly associated with the presence of CMBs in this study. Furthermore, because the effect of diabetes itself without hypertension on small vessel diseases in the brain remains controversial, ${ }^{14}$ the discrepancy between the univariate and multivariate analysis results showed that diabetes itself might not independently influence the existence of CMBs. In contrast to previous studies on WMH in the Gangnam survey (from a different center), ${ }^{15,16}$ we measured WMH volume rather than using visual rating scales. Using this strategy, we could obtain a more sensitive and objective cut-off value for WMH in the younger population. Moreover, our study did not show a difference in CMB presence with respect to total cholesterol level, which was in contrast to previous studies. ${ }^{17,18}$ We assume that this was based on population discrepancy because our research was subject to neurologically healthy patients.

This study has a few caveats that require careful consideration. First, since our study was a cross-sectional, observational evaluation for aspirin use with CMBs, longitudinal studies with follow-up data for CMBs are required to evaluate the causal relationship between them. Second, in subjects with vascular risk factors but without a history of clinical stroke, aspirin is the most popular medication for the primary prevention of stroke in South Korea. Therefore, it was challenging to gather information of other types of antithrombotics in our study population. Third, as we acquired data from self-reported questionnaires, there is an uncertainty in ascertaining the exact medical history, especially for investigating indications of aspirin use. In a future prospective study, the effects of other antithrombotics and aspirin may be considered.

The association between antiplatelet use and CMBs remains controversial. Considering the notable side effects of antithrombotic use, vigorous efforts are required to identify intermediary radiological and clinical markers such as CMBs and WMH with regard to intracranial hemorrhage.

\section{Acknowledgments}

This research was supported by the Research Program 2017 funded by Seoul National University College of Medicine Research Foundation and the R\&D support grant (O2001021) of Korea University Guro Hospital, Republic of Korea.

\section{Conflicts of interest}

No potential conflicts of interest relevant to this article was reported.

\section{REFERENCES}

1. Kim BJ, Lee SH. Cerebral microbleeds: their associated factors, radiologic findings, and clinical implications. J Stroke. 
2013;15:153-163.

2. Koennecke HC. Cerebral microbleeds on MRI: prevalence, associations, and potential clinical implications. Neurology. 2006;66:165-171.

3. Kim CK, Kwon HT, Kwon HM. No significant association of aspirin use with cerebral microbleeds in the asymptomatic elderly. J Neurol Sci. 2012;319:56-58.

4. Nishikawa T, Ueba T, Kajiwara M, Miyamatsu N, Yamashita K. Cerebral microbleeds in patients with intracerebral hemorrhage are associated with previous cerebrovascular diseases and white matter hyperintensity, but not with regular use of antiplatelet agents. Neurol Med Chir (Tokyo). 2009;49:333-338; discussion 338-339.

5. Ge L, Niu G, Han X, Gao Y, Wu Q, Wu H, et al. Aspirin treatment increases the risk of cerebral microbleeds. Can J Neurol Sci. 2011;38:863-868.

6. Liu S, Li C. Antiplatelet drug use and cerebral microbleeds: a meta-analysis of published studies. J Stroke Cerebrovasc Dis. 2015;24:2236-2244.

7. Vernooij MW, Haag MD, van der Lugt A, Hofman A, Krestin GP, Stricker BH, et al. Use of antithrombotic drugs and the presence of cerebral microbleeds: the Rotterdam Scan Study. Arch Neurol. 2009;66:714-720.

8. Lovelock CE, Cordonnier C, Naka H, Al-Shahi Salman R, Sudlow CL, Edinburgh Stroke Study G, et al. Antithrombotic drug use, cerebral microbleeds, and intracerebral hemorrhage: a systematic review of published and unpublished studies. Stroke. 2010;41:1222-1228.

9. Pantoni L. Cerebral small vessel disease: from pathogenesis and clinical characteristics to therapeutic challenges. Lancet Neurol. 2010;9:689-701.
10. Kim BJ, Lee SH. Prognostic impact of cerebral small vessel disease on stroke outcome. J Stroke. 2015;17:101-110.

11. Altamura C, Scrascia F, Quattrocchi CC, Errante Y, Gangemi E, Curcio G, et al. Regional MRI diffusion, white-matter hyperintensities, and cognitive function in Alzheimer's disease and vascular dementia. J Clin Neurol. 2016;12:201-208.

12. Lee JE, Shin DW, Yun JM, Kim SH, Nam YS, Cho B, et al. Insulin resistance is a risk factor for silent lacunar infarction. Stroke. 2016;47:2938-2944.

13. Kwon HM, Park JH, Park JH, Jeong HY, Lim JS, Jeong HG, et al. Visceral fat is an independent predictor of cerebral microbleeds in neurologically healthy people. Cerebrovasc Dis. 2016;42:90-96.

14. Caplan LR. Lacunar infarction and small vessel disease: pathology and pathophysiology. J Stroke. 2015;17:2-6.

15. Kim CK, Lee SH, Kim BJ, Ryu WS, Yoon BW. Age-independent association of pulse pressure with cerebral white matter lesions in asymptomatic elderly individuals. J Hypertens. 2011;29:325-329.

16. Kim CK, Lee SH, Kim BJ, Ryu WS, Choi SH, Oh BH, et al. Elevated leukocyte count in asymptomatic subjects is associated with a higher risk for cerebral white matter lesions. Clin Neurol Neurosurg. 2011;113:177-180.

17. Lee SH, Bae HJ, Kwon SJ, Kim H, Kim YH, Yoon BW, et al. Cerebral microbleeds are regionally associated with intracerebral hemorrhage. Neurology. 2004;62:72-76.

18. Lee SH, Bae HJ, Yoon BW, Kim H, Kim DE, Roh JK. Low concentration of serum total cholesterol is associated with multifocal signal loss lesions on gradient-echo magnetic resonance imaging: analysis of risk factors for multifocal signal loss lesions. Stroke. 2002;33:2845-2849. 